\title{
A NOVEL APPROACH FOR LONG TERM SOLAR RADIATION PREDICTION
}

\author{
Manju Khanna ${ }^{1}$, N.K. Srinath ${ }^{2}$ and J.K. Mendiratta ${ }^{3}$ \\ Department of Computer Science and Engineering, R. V. College of Engineering, India
}

\begin{abstract}
With present stress, being laid on green energy worldwide, harnessing solar energy for commercial use has importance in sizing and longterm prediction of solar radiation. However, with continuous changing environment parameters, it is quite difficult for long-term prediction of solar radiation. In the past research scholars, have carried out solar prediction only for a few days, which is insufficient to exploit the radiation for sizing and harnessing the solar energy for commercial use. To overcome this gap, present work utilizes application of lifting wavelet transform along with ANFIS to predict the radiation for long duration.
\end{abstract}

Keywords:

Statistical Methods, ARIMA, RNN, Wavelet Transform, MRA, NeuroFuzzy Inference System, RMSE

\section{INTRODUCTION}

For sizing of Photovoltaic (PV) configuration, connected either in isolation or with power grid, total solar radiation forms an important parameter [1], [2]. For commercial planning and sizing of a PV system long term forecasting is required. Different forecasting methods are available, which can be categorized as (i) Physical, (ii) Statistical and (iii) Hybrid. The physical models produce good results for long term forecasting, and use data produced through Numerical Weather Prediction (NWP) along with the satellite imagery. NWP models require physics of the atmosphere, using current information therefore require super computers to forecast for long term. The statistical models are mathematical and require historical data for prediction. These models are further categorized (i) persistence (ii) time series models, both of them use Auto Regressive (AR), Moving Average (MA) or Auto Regressive Moving Average (ARMA) algorithms. The persistence model, predicts the value by assuming future value as same as the present one and so the simplest in computation. On the other hand, time series models use historic data, considered as a sequence measured over time. The Hybrid models are combination of the above two models and are named combined models.

In the past research scholars have made efforts in prediction of solar radiation for a maximum duration of one or two days. This duration of prediction is not sufficient to exploit the solar energy at commercial level and planning of energy storage devices. The energy storage devices feed power to the grid, in case of peak load demand or compensated for sudden requirement, which occurs, with change of environment conditions. To meet the above gap for planning and investment on the part of energy service providers, the research work carried out shall be of immense use. Solar energy is a source of green energy, which fluctuates with time of the day, season and place, and consists of time series data taken at regular intervals. In addition, it is loaded with noise in measurements, and radiation conditions. The past efforts in prediction of solar radiation carried out by application of statistical methods, such as ARMA, AR, ARIMA and other methods, which yielded more error with increase in duration of prediction, so not suitable for the purpose cited. Some techniques of data reduction also tried, which include Singular Value decomposition (SVD), Principal Component Analysis (PCA) etc., but could not produce long-term prediction. Some scholars tried to combine different techniques for better performance, but with not much success.

In view of above gap, efforts made by application of fast Fourier Transform (FFT), Discrete Wavelet Transform (DWT) for feature extraction along with other techniques mentioned, which found to consume more of computer resources.

In view of above gap, efforts made by application of fast Fourier Transform (FFT), Discrete Wavelet Transform (DWT) for feature extraction along with other techniques mentioned, which found to consume more of computer resources. With development of Lifting Wavelet Transform, when implemented needed no extra computer resources along with a simple algorithm. Further, the algorithm filters out the noise loaded with the data, thereby producing much better performance. The feature-extracted data produces two parts of wavelet coefficients, reducing data by a power of 2 thereby providing fast and efficient data reduction. The technique of Lifting Wavelet Transform (LWT) made possible to predict the solar radiations for a long term, i.e. up to three months, which was found to meet the requirements of energy service provider.

The contribution of the proposed work is as follows:

- The research scholars have utilized Lifting Wavelet Transforms along with other techniques for feature extraction for solar radiation prediction. Effort made by them used only the multi-level analysis at one level and combined it along with data mining algorithms.

- The Present work contributed to feature extraction of solar radiation by application of Lifting Wavelet Transform at multi-level, thereby reducing the data up to one eight level, thereby making it possible to handle for a long duration. It is shown by the authors that a reduction of data up to third level of resolution provide the best results. In view of the results provided, the feature extraction carried out accordingly.

- The authors have developed and implemented the algorithm for multi-level resolution and demonstrated with different applications in feature extraction of time series data.

- The algorithm analyzed and developed for clustering of data, rejecting lower values of wavelet transform. The algorithm made possible to predict for solar radiation for long duration, producing quite accurate results. The ANFIS algorithm tried on various types of membership function, with their performance comparison and the one giving better results selected for long-term prediction. Thus, the contribution 
helped in long-term prediction of any time series application, which can be exploited for varied applications.

- The algorithm developed is implemented for solar radiation for long term, such as for one month and for three months duration prediction and results presented. The results obtained with actual and with featured data found to be quite accurate, thereby justifying and validation of the algorithm.

The paper introduces the requirement of the work carried out in the first part, section 2 includes the related work. Section 3 introduces basics involved in the work, giving an overview of wavelets, requirement of second-generation wavelets, including Lifting Wavelet Transform, multi resolution analysis with their properties. The analysis helped in generation of multi-level Lifting wavelet transforms. Section 4 deals with construction of multi-level lifting wavelet transform, needed for design of algorithm, basics of ANFIS algorithm with its different layers to obtain long-term prediction of solar radiation. Section 5 describes the experiments carried out on solar radiation data, obtained from NREL site, giving plots of prediction for one month and for three months. Different variety of Membership Functions (MFs) tried, a performance comparison done and the one performing best selected. Last section deals with conclusion drawn and applicability of the algorithm suggested for time series data.

\section{RELATED WORK}

Solar radiations are stochastic in nature with random observations of data. In the past many research scholars have made efforts in prediction of solar radiation by application of Auto Regressive (AR) [3], and AR integrated (ARIMA) [4] models. These models depend upon simplification of statistical assumptions about measured data, producing relatively large errors, and so are not suitable for practical applications. Joao Paulo Coelho at el have applied Support Vector Regression (SVR), Autoregressive Hidden Markov Model (HMM) and a hybrid technique, in combination with both SVR and Markov Chain [5] for a temperature control application. The best results shown are with a combination of SVR and Markov Chain models. Prema and Rao [6] tried different statistical models by taking time series data of solar radiation and predicted one day ahead. They found application of Moving Average (MA) method to produce better results by taking time series data. Many scholars have applied recurrent neural networks (RNNs) to time series data [7] [8] [9]. Capizzi et al. [10] have exploited wavelets along with recurrent neural network for solar radiation prediction by using observed meteorological data. The duration of predicted data of solar radiation is limited to only two days [10]. Even the commercial packages, using ANN produce prediction for a duration of 10 days [11]. Olatomiwa et al. [12] utilized a Support Vector Machine firefly algorithm for horizontal global solar radiation prediction and found to have more accurate results compared to Artificial Neural Network and Genetic programming models. Data used by them comprised of Maximum temperature, Minimum temperature and sun shine duration from meteorological parameters, with an average prediction range of one month. Recently, Al-Shou and Alawasa [13] have used Nonlinear Autoregressive Recurrent Neural Networks (NARX) Neural Networks Model for solar radiation prediction. The meteorological parameters included by them for the model consisted of temperature, wind speed and humidity data for oneyear duration. The data taken for the above parameters taken at an interval of one hour, as a time series data, but by taking the actual data without application of any feature extraction technique. Similar efforts made by Aziz Ahmad and Tim Anderson [14] for solar radiation prediction using weather variables of temperature (maximum and minimum), Air pressure, humidity, Solar Zenith angle, Azimuth angle, rain amount, wind speed and wind direction. They also used NARX models by application of different number of neurons and layers and found the results much superior compared to previous different models used by other researchers.

Above literature, survey reveals that though recently some effort has been made for long duration solar radiation prediction using ANN, the data was taken from metrological information. However, present work carried out for sizing and planning of solar radiation by utilizing Lifting Wavelet Transform for feature extraction of the time series data, along with application of Artificial Neural Fuzzy Inference System (ANFIS). The ANFIS system utilizes knowledge of an expert as in fuzzy system and extrapolating it by using the neural networks for long-term prediction of solar radiation.

\section{OVERVIEW OF BASICS}

A brief overview of basics required for long-term solar radiation prediction as below:

\subsection{WAVELETS}

The wavelets are thought data building blocks, allowing representation efficiently with fast computing, through dyadic translation and dilation of a function, i. e. $\psi_{j, m(x)}=\psi\left(2^{j} x-m\right)$, named as first generation wavelets [15], with properties:

1. Creates a Riesz basis in $L_{2}(R)$ with a variety of function spaces $\mathcal{F}$.

2. Coordinate function $\bar{\Psi}_{j, m}$, denoted by $\gamma_{j, m}=: \bar{\Psi}_{j, m}(f)$ generates orthogonal or duals (bi-orthogonal) wavelets.

3. They and their duals generate vanishing polynomial moments in local space and generate frequency, which decays towards slow frequencies.

4. Create multi-resolution analysis, leading to fast wavelet transform, allowing passing the function $f$ along with its coefficients $\gamma_{j, m}$ in a linear time.

Above features have been exploited in varied applications [15]. By generalization, these wavelets not only translate and dilate, but also provide:

1. Bases solution to differential and integral equations not only on $R^{n}$, but also on arbitrary, non-smooth domains of $R^{n}$ also.

2. Whereas these wavelets provide bases for spaces with translation invariants, the second-generation wavelets make them suitable for differential functions and analysis on curved surfaces with weighted approximation.

3. Provide solution for irregular sampled data. 
Thus, generalization of the first generation wavelets, preserves its properties but also above provides additional properties, therefore named second-generation wavelets.

\subsection{REQUIREMENT OF SECOND GENERATION WAVELETS (LIFTING SCHEME) [16]}

Several algorithms developed for construction of wavelets for functions on intervals [17] - [20], bounded domains [21], solution for irregular samples [22], [23]. However, lifting scheme provides a simple technique to construct second-generation wavelets. A multi-resolution analysis working upwards is applied to achieve desired properties of custom designed filters. However, a check is required on these filters for generating functions with a stable basis for smoothness of the wavelets.

\subsection{MULTI-RESOLUTION ANALYSIS (MRA) [23]}

For closed subspaces, a wavelet generates direct sum of decomposition in $L_{2}(R)$ :

$$
V_{j}=\ldots+W_{j-2}+W_{j-1}, \quad j \in \mathrm{Z} \text { in } L_{2}(R)
$$

where, + Indicates direct sums of the components. For an orthogonal function $f$, its decomposition is mutually orthogonal with the subspaces:

$$
\begin{gathered}
\ldots \subset V_{-1} \subset V_{0} \subset V_{1} \ldots \\
\operatorname{clos}_{2}\left(\bigcup_{j \in Z} V_{j}\right)=L_{2}(R) \\
\bigcap_{j \in Z} V_{j}=\{0\} ;
\end{gathered}
$$

A domain $V_{j} \quad j \in \mathrm{Z}$, with a Riesz basis, generates a scaling functions $\left\{\phi_{j, k} \mid k \in K(j)\right\}$ with $K(j)$ forming a general set of index. With the above properties, if a single function subspace $V_{0}$ generated by $\varphi \in L_{2}(R)$ with:

$$
V_{0}=\operatorname{clos}_{L_{2}(R)}\left\langle\varphi_{0, k}: k \in Z\right\rangle
$$

where, clos defining a closed domain.

A function $\varphi_{j, k}(x) \equiv 2^{j / 2} \varphi\left(2^{j} x-k\right)$, generating subspaces $V_{j}$ by itself, just as $W_{j}$ generated by function $\psi$ with $W_{j} \equiv \operatorname{clos}_{L_{2}(R)}$ $\left\langle\psi_{j, k}: k \in Z\right\rangle$ with duals of $\varphi$ as $\bar{\varphi}$ and of $\psi$ as $\bar{\psi}$ satisfying:

$$
\left\langle\psi_{j, k} \bar{\psi}_{l, m}\right\rangle=\delta_{j, l} \cdot \delta_{k, m} \quad j, k, l, m \in \mathrm{Z}
$$

From above analysis, defining:

Definition 1: A function $\varphi \in L_{2}(R)$ generates a Multi Resolution Analysis (MRA), producing a nested sequence of closed subspaces $V_{j}$, satisfying properties (1), (2), (3) and (4), thereby $\left\{\varphi_{0, k}\right\}$ forms a Riesz basis of $V_{0}$.

From above definition, taking two constants $A$ and $B$ with $0<A \leq B<\infty$, such as

$$
A\left\|\left\{c_{k}\right\}\right\|_{l^{2}}{ }^{2} \leq\left\|\sum_{k=-\infty}^{\infty} c_{k} \varphi_{0, k}\right\|_{2}^{2} \leq B\left\|\left\{c_{k}\right\}\right\|_{l^{2}}{ }^{2}
$$

Generates bi-infinite square sum able sequences $\left\{c_{k}\right\}$, i. e.

$$
\left\|\left\{c_{k}\right\}\right\|_{l^{2}}^{2}=\sum_{k=-\infty}^{\infty}\left|c_{k}\right|^{2}<\infty
$$

A function $\varphi$ generating an MRA, defined as a scaling function.

\section{CONSTRUCTION OF LWT}

For construction of these wavelets, two things are required: (i) a set of partitioning and (ii) a filter. A finite filter in context of MRA defined [23], [27]:

$$
\left\{h_{j, k, l} \mid j \in \mathrm{Z}, k \in K(j), l \in K(j+1)\right\}
$$

(i) for $j$ and $k$ coefficients $h_{j, k}$, have a non-zero finite number set:

$$
L(j, k)=\left\{l \in K(j+1) \mid h_{j, k, l} \neq 0\right\}
$$

(ii) Similarly for $j$ and $l$, coefficients $h_{j, k, l}$ have a non-zero finite number set:

$$
K(j, l)=\left\{k \in K(j) \mid h_{j, k, l} \neq 0\right\},
$$

(iii) For all $j, k, l$ sets $L(j, l)$ and $K(j, l)$ are uniformly bounded.

Taking a signal $s$ of a finite sequence $L, a_{0}=\left(a_{0, n}\right)(n=0,1, \ldots$, $L-1)$, characterizing the signal.

Defining the sequences $a_{1}=\left(a_{1, n}\right)$ and $d_{1}=\left(d_{1, n}\right)$ :

$$
a_{(m+1), n}=\sum_{k=2 n}^{2 n=N} h_{k-2 n} a_{m k}
$$

where, $n=\frac{L}{2}-1$ for $L$ as even number and $n=\frac{L-1}{2}$ for $L$ as odd number.

Also,

$$
d_{(m+1) n}=\sum_{k=2 n+1-N}^{2 n+1}(-1)^{k} h_{2 n+1-k} a_{m k}
$$

where, $n=\frac{L+N-3}{2}$ for $L$ as even number and $n=\frac{L+N-2}{2}$ for $L$ as odd number.

From above equations, with $m=0, a_{(m+1), n}$ will consist of half the length of $a_{0}$, with decomposition:

$$
a_{0 n}=\sum_{k=-\infty}^{\infty}\left[h_{n-2 k} a_{1 k}+(-1)^{n} h_{2 k+1-n} d_{1 k}\right]
$$

where, $a_{1}$ and $d_{1}$ can be reconstructed precisely for every $n \in \mathrm{Z}$ [26]. For a finite vector sequence $p=\left(p_{n}\right)$, defining energy $\|p\|^{2}$ by,

$$
\|p\|^{2}=\sum_{n=-\infty}^{\infty} p_{n}^{2}
$$

With square root $\|p\|$ with $\|p\|_{2}$ called a Euclidean norm. Thereby, $a_{0}, a_{1}, d_{1}$ will satisfy:

$$
\left\|a_{0}\right\|^{2}=\left\|a_{1}\right\|^{2}+\left\|d_{1}\right\|^{2}
$$

A recurring application of the above equations generates $a_{0}$ into a matrix form with a sequence of $d_{1}, d_{2}, \ldots, d_{M}, a_{M}$, shown in Fig.1. 
These sequences possess the same amount of information as $a_{0}$,

$$
\left\|a_{0}\right\|^{2}=\left\|a_{M}\right\|^{2}+\sum_{m=1}^{M}\left\|d_{m}\right\|^{2}
$$

Thus, wavelet coefficients of the signal $s$, in the form of above matrix are reduced by a power of 2 at each level. Thus, depending upon the size of the signal samples, feature extraction of the signal carried out. The $a_{M}$ coefficients represent approximate of signal features, while various $d_{M}$ components the details of the signal features.

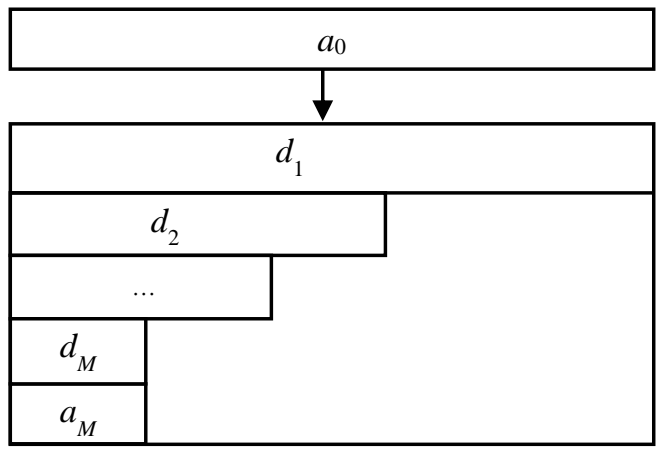

Fig.1. LWT of a Sequence $a_{0}$

\subsection{NEURO-FUZZY INFERENCE SYSTEM [27]}

They are equivalent to fuzzy inference systems, with adaptive networks, thereby named as Adaptive Neuro Fuzzy Inference System (ANFIS), organically blending fuzzy logic and neural network. It follows with Sugeno Fuzzy model, also known as Takagi, Sugeno and Kang (TSK) Fuzzy model [29], and provides a symmetric method to produce fuzzy rules.

Architecture: A Sugeno fuzzy model with inputs $x, y$ and an output $z$, shall comprise of:

- If $A_{1}$ defined by $x$ and $B_{1}$ defined by $y$ then

$$
f_{1}=p_{1} x+q_{1} y+r_{1}
$$

- Similarly, if $A_{2}$ defined by $x, B_{2}$ defined by $y$, then

$$
f_{2}=p_{2} x+q_{2} y+r_{2}
$$

The typical configuration of above rules shown in the Fig.2.

Layer 1: Taking $i$ as adaptive nodes represented by a function:

$$
\begin{gathered}
O_{1, i}=\mu_{A i}(x), \text { for } i=1,2 \\
\text { or } \\
O_{1, i}=\mu_{B i-2}(y), \text { for } i=3,4
\end{gathered}
$$

With $x$ or $y$, as input to node $i$ with $A_{i}$ or $B_{i-2}$ being linguistic labels for the node. $O_{1, i}$ as a membership grade of a fuzzy set $A$, specifying the degree of the input $x$ (or $y$ ) as a quantifier of $A$.

Layer 2: all nodes fixed and labeled as $\pi$, indicating a product of the incoming signals:

$$
O_{2, i}=w_{i}=\mu_{A i}(x) \mu_{B i}(y), i=1,2
$$

Each output node representing the firing strength of a rule.

Similarly, Layer 3 has all the fixed nodes denoted by $N$, with the $i^{\text {th }}$ node-calculating ratio of firing strength of the $i^{\text {th }}$ rule:

$$
O_{3, i}=\bar{w}_{i}=\frac{w_{i}}{w_{1}+w_{2}}, i=1,2
$$

Layer 4: As in layer 1, all $i$ nodes adaptive and denoted by a function:

$$
O_{4, i}=\bar{w}_{i} f_{i}=\bar{w}_{i}\left(p_{i} x+q_{i} y+r_{i}\right)
$$

$\bar{w}_{i}$ being a normalized firing strength from layer 3 with $\left\{p_{i}, q_{i}, r_{i}\right\}$ as the parameters of the node.

Layer 5: A node labeled $\Sigma$, computes overall output of all the incoming signals,

$$
O_{5, i}=\sum_{i} \bar{w}_{i} f_{i}=\frac{\sum_{i} w_{i} f_{i}}{\sum_{i} w_{i}}
$$

Depending upon the requirements, the layers 3 and 4 can be combined, with a four-layer network. Similarly, normalization of weights carried out in the last layer.

To determine initial ANFIS architecture, structure identification is required, which involves (1) Selection of relevant input variables (2) Determining an initial ANFIS architecture, which includes:

- Partitioning of Input space

- Membership functions required

- Fuzzy if-then rules required

- Antecedent and Consequent parts

- Selection of initial parameters for MFs.

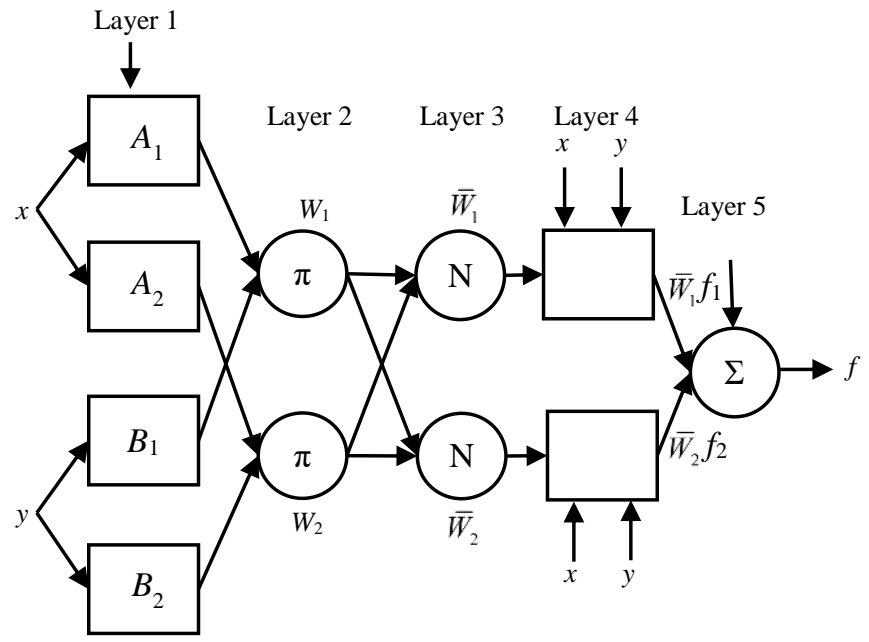

Fig.2. Typical configuration of ANFIS

Mean Square Error (MSE): A convenient method for measuring an average error indicating the degree of data change and denoted by:

$$
M S E=\frac{1}{n} \sum_{t=1}^{n}\left(y_{t}-\hat{y}_{t}\right)^{2}
$$

With $y_{1}, y_{2}, \ldots y_{t}$ being the actual observations and $\hat{y}_{1}, \hat{y}_{2} \ldots \hat{y}_{t}$ the predicted variables.

Whereas the Root Mean MSE (RMSE) provides a square root value of MSE and is given by,

$$
\mathrm{RMSE}=\sqrt{\sum_{k=1}^{t} \frac{\left(\hat{O}_{k}-O_{k}\right)^{2}}{t}}
$$


With $O$ being the output function and $\hat{O}$, the estimated function.

\section{EXPERIMENT}

For experiment purpose, radiation data from NREL-USA site used. The performance of prediction algorithm with ANFIS for the original data and by using Lifting wavelet transform along with ANFIS carried out. The original data has been reduced to one third level of Lifting wavelet transform, thus carrying out feature extraction and filter of the original data, thereby reducing the data to one eight of the actual data. Reduction of data also reduces requirement of resources in the computer. The ANFIS algorithm has been tried with triangle, bell and trapezoidal membership functions (MFs) with plots of original and features extracted data, using Lifting Wavelet transform. The performance compared for all the MFs mentioned.
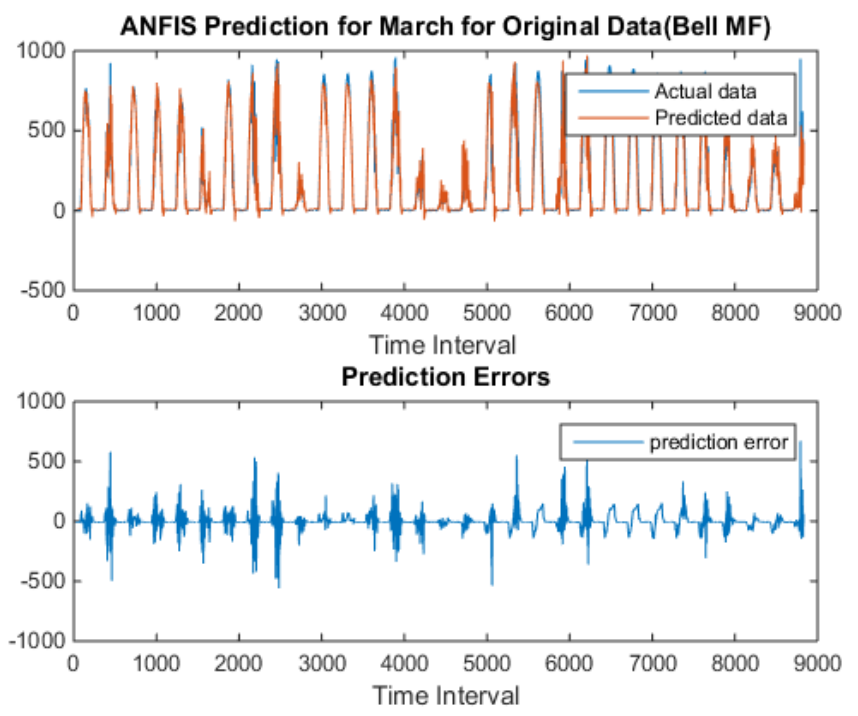

Solar Time Series and ANFIS Prediction for March using LWT(Bell MF)
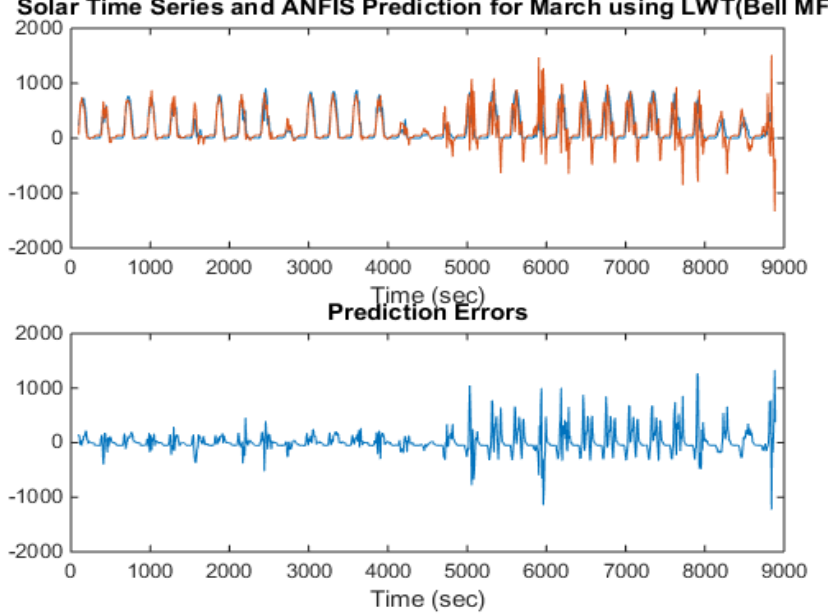

Fig.3. Plot of prediction and Error for actual and data with Lifting Wavelet Transform and with Trapezoidal MF for one month

The Fig.3 shows one-month prediction, i.e. by taking data for the month of February and predicting for the month of March. First part of the Fig.3 represents actual data in 'blue' color while plot in 'red' color shows the predicted values for the same period, by using 'bell MF' for the ANFIS system. Similarly, third part of the Fig. 3 shows plots for featured data for same period with 'bell MF' and the fourth part of the Fig.3 shows error values when featured data applied for prediction for one month duration. The simulation studies carried out for the above actual and featured data validate the ANFIS system.

Further, the data has been reduced by one eight, thereby creating a big gap of data taken, shows the predicted error at the far end of the data more as compared to actual data. However, modeling of the training data was carried with Trapezoidal, Bell and Triangular MFs, for original and featured data for one month and RMSE error for all the three MFs is compared in the Fig.4.
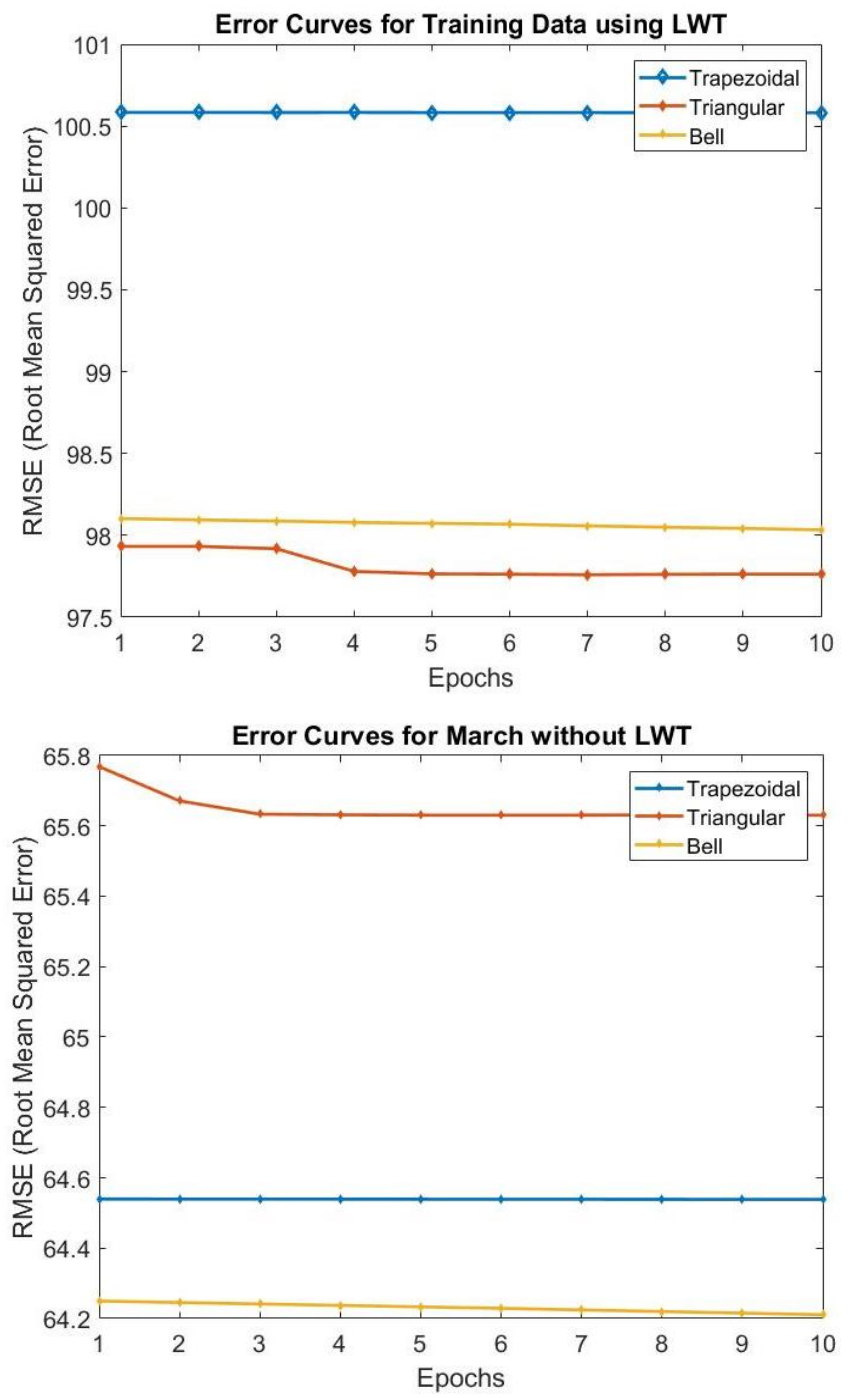

Fig.4. RMSE error curves for one-month data and extracted featured data

From Fig.4 and Table.1, we find that Bell MF performs better as compared to Trapezoidal and Triangle MFs. The Fig.5 below gives error plots for three months duration:

It is evident from Fig.5 above that the similar results for threemonth duration prediction are found. There is not much difference in error values for three months prediction with original and with Lifting wavelet reduced and filtered data. Further, these results also show that there is not much difference in performance of 
results for actual and featured data simulation. However, with reduced data, resources required are reduced along with fast computation of the results.

From the above plots in Fig.4 and Table.1, we find that the performance with triangle and trapezoidal MFs is poor compared to bell MF. Similar case found for three months of actual and with featured extracted data, which is shown in Fig.6.

Plot of training for the three MFs with actual and featured data, shows the performance with Bell MF function is better compared to other MFs. In addition, triangle MF shows overfitting of the data, so it is rejected. Trapezoidal MF function performs better compared to Triangle MF. However, Bell MF gives less error, similar to one-month performance, so it is selected for long-term prediction. Further, as mentioned above, performance of featured data is better as compared to that with actual for three months. It is concluded that more data helps in better training and learning of the system.

Solar Time Series and ANFIS Prediction for May,June,July(Original)
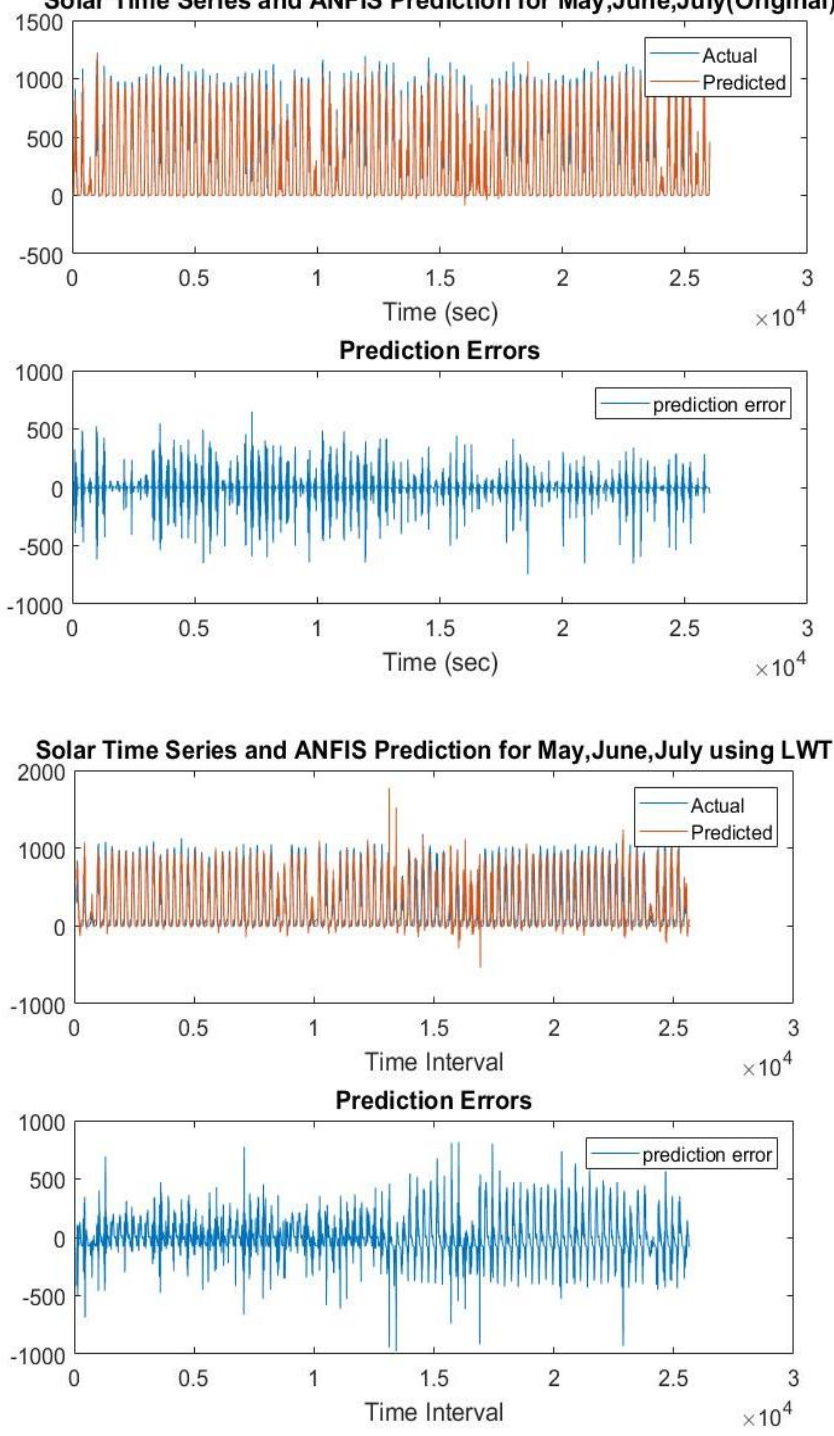

Fig.5. Plot of prediction and Error for actual and data with Lifting Wavelet Transform and with Trapezoidal MF for three month
Table.1. Performance comparison for original and featured data for one month

\begin{tabular}{|c|c|c|c|c|}
\hline Data & Data Type & $\begin{array}{c}\text { Triangle } \\
\text { MF }\end{array}$ & Bell MF & $\begin{array}{c}\text { Trapezoidal } \\
\text { MF } \\
\end{array}$ \\
\hline \multirow{2}{*}{$\begin{array}{l}\text { Original } \\
\text { Data }\end{array}$} & $\begin{array}{l}\text { Training data } \\
\text { Performance } \\
\text { (RMSE) }\end{array}$ & $\begin{array}{l}4400 \\
65.76\end{array}$ & $\begin{array}{l}4400 \\
64.24\end{array}$ & $\begin{array}{l}4400 \\
64.53\end{array}$ \\
\hline & $\begin{array}{l}\text { Testing data } \\
\text { Performance } \\
\text { (RMSE) }\end{array}$ & $\begin{array}{l}4400 \\
78.53\end{array}$ & $\begin{array}{l}4400 \\
74.88\end{array}$ & $\begin{array}{l}4400 \\
77.35\end{array}$ \\
\hline \multirow{2}{*}{$\begin{array}{l}\text { LWT } \\
\text { Data }\end{array}$} & $\begin{array}{l}\text { Training data } \\
\text { Performance } \\
\text { (RMSE) }\end{array}$ & $\begin{array}{c}550 \\
97.93\end{array}$ & $\begin{array}{c}550 \\
98.10\end{array}$ & $\begin{array}{c}550 \\
100.5\end{array}$ \\
\hline & $\begin{array}{l}\text { Testing data } \\
\text { Performance } \\
\text { (RMSE) }\end{array}$ & $\begin{array}{c}550 \\
124.6 \text { to } \\
705.0\end{array}$ & $\begin{array}{c}550 \\
144.3 \text { to } \\
144.1\end{array}$ & $\begin{array}{c}550 \\
143.5\end{array}$ \\
\hline
\end{tabular}
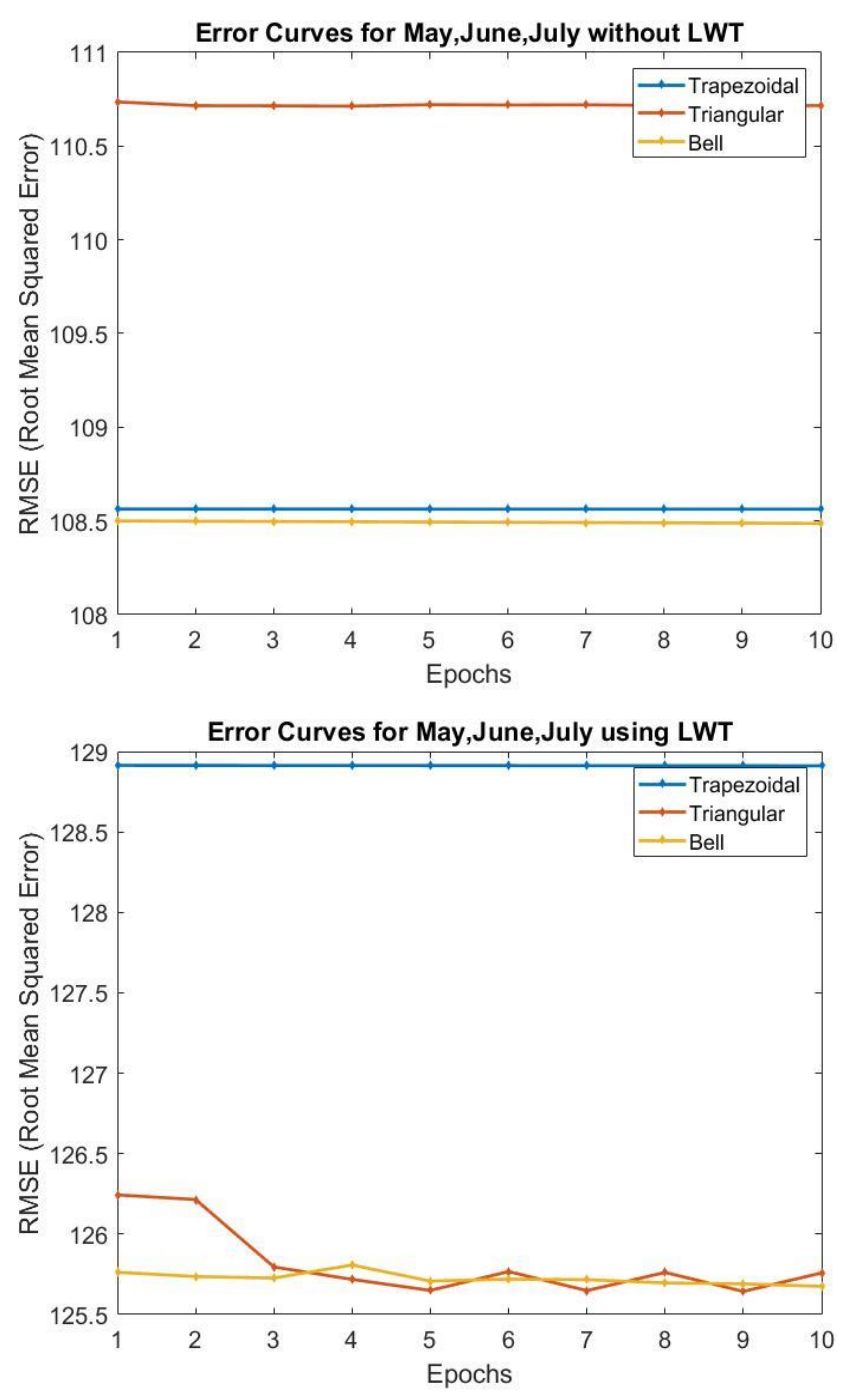

Fig.6. Performance of training data for three months without and with featured data by LWT 
Table.2. Performance comparison for original and featured data for three months

\begin{tabular}{|c|c|c|c|c|}
\hline Data & Data Type & $\begin{array}{c}\text { Triangle } \\
\text { MF }\end{array}$ & Bell MF & $\begin{array}{c}\text { Trapezoidal } \\
\text { MF }\end{array}$ \\
\hline \multirow{2}{*}{$\begin{array}{c}\text { Original } \\
\text { Data }\end{array}$} & $\begin{array}{l}\text { Training data } \\
\text { Performance } \\
\text { (RMSE) }\end{array}$ & $\begin{array}{l}13000 \\
110.73\end{array}$ & $\begin{array}{l}13000 \\
108.5\end{array}$ & $\begin{array}{l}13000 \\
108.56\end{array}$ \\
\hline & $\begin{array}{l}\text { Testing data } \\
\text { Performance } \\
\text { (RMSE) }\end{array}$ & $\begin{array}{l}13000 \\
131.52\end{array}$ & $\begin{array}{l}13000 \\
132.93\end{array}$ & $\begin{array}{c}13000 \\
131.737\end{array}$ \\
\hline \multirow{2}{*}{$\begin{array}{l}\text { LWT } \\
\text { Data }\end{array}$} & $\begin{array}{c}\text { Training data } \\
\text { Performance } \\
\text { (RMSE) }\end{array}$ & $\begin{array}{c}1600 \\
126.2 \text { to } \\
125.7\end{array}$ & $\begin{array}{c}1600 \\
125.76\end{array}$ & $\begin{array}{c}1600 \\
128.91\end{array}$ \\
\hline & $\begin{array}{c}\text { Testing data } \\
\text { Performance } \\
\quad(\mathrm{RMSE})\end{array}$ & $\begin{array}{c}1600 \\
159.93 \text { to } \\
8830.87\end{array}$ & $\begin{array}{c}1600 \\
173.10 \text { to } \\
173.01\end{array}$ & $\begin{array}{c}1600 \\
175.55\end{array}$ \\
\hline
\end{tabular}

\section{CONCLUSION}

The existing literature shows that though efforts have been made for long-term solar radiation prediction using various algorithm, but present technique employs a simple method in prediction for a long duration. A time series data suffers from socalled 'curse of dimensionality' and is often loaded with noise, thereby requires feature extraction and filtering of the data. These requirements are taken care of by application of Lifting Wavelet Transform along with ANFIS. The results presented show good results for a long-term prediction. With varied applications generating time series data, the method developed shall be well suited and so can be put to use for commercial utilization.

\section{REFERENCES}

[1] M. Egido and E. Lorenzo, "The Sizing of Standalone PVSystem: A Review and a Proposed New Method", Solar Energy Materials and Solar Cells, Vol. 26, No. 1, pp. 51-69, 1992.

[2] Mukund R. Patel, "Wind and Solar Power Systems-Design, Analysis and Operation", $2^{\text {nd }}$ Edition, CRC Press, 2006.

[3] S. Hokoi, M. Matsumoto and M. Kagawa, "Stochastic Models of Solar Radiation and Outdoor Temperature", Proceedings of International Conference on Society of Heating, Refrigerating and Air Conditioning Engineers, pp. 10-17, 1990.

[4] L. Mora-Lopez and M. Sidrach-De-Cardoma "Multiplicative ARMA Models to Generate Hourly Series of Global Irradiation", Solar Energy, Vol. 63, No. 5, pp. $283-$ 291, 1998.

[5] Joao Paulo Coelho and Jose Boaventura-Cunha, "Long Term Solar Radiation Forecast using Computational Intelligence Methods", Applied Computational Intelligence and Soft Computing, Vol. 2014, pp. 1-14, 2014.

[6] V. Prema and K. Uma Rao, "Development of Statistical Time Series Models for Solar Power Prediction", Renewable Energy, Vol. 83, pp. 100-109, 2015.
[7] W. Yan, "Toward Automatic Time Series Forecasting using Neural Networks", IEEE Transactions on Neural Networks and Learning Systems, Vol. 23, No. 7, pp. 1028-1039, 2012.

[8] M. Qi and G.P. Zhang, "Trend Time Series Modeling and Forecasting with Neural Networks", IEEE Transactions on Neural Networks, Vol. 19, No. 5, pp. 808-816, 2008.

[9] R.V. Borges, A.A. Garez and L.C. Lamb, "Learning and Representing Temporal Knowledge in Recurrent Networks", IEEE Transactions on Neural Networks, Vol. 22, No. 12, pp. 2409-2421, 2011.

[10] G. Capizzi, C. Napoli and F. Bonanno, "Innovative Second generation Wavelets Construction with Recurrent Neural networks for Solar radiation Forecasting", IEEE Transaction on Neural networks and Learning Systems, Vol. 23, No. 11, pp. 1805-1815, 2012.

[11] Solar Power Forecast for up to 10 Days Ahead, Available at: solargis.com/products/forecast/overview.

[12] David L. Donoho, "Unconditional Bases are Optimal Bases for Data Compression and for Statistical Estimation", Journal of Applied and computer. Harmonic Analysis, Vol. 1, No. 1, pp. 100-115, 1993.

[13] Yazeed A. Al-Sboul and Khaled M. Alawasa, "Nonlinear Autoregressive Recurrent Neural Network Model for Solar Radiation Prediction", International Journal of Applied Engineering Research, Vol. 12, No. 14, pp. 4518-4527, 2017.

[14] Aziz Ahmad and Tim Anderson, "Global Solar Radiation Prediction using Artificial Neural Network Models for New Zealand", Proceedings of IEEE 52 ${ }^{\text {nd }}$ Annual Conference of the Australian Solar Council, pp. 12-16, 2014.

[15] D.L. Donoho, "Unconditional Bases are Optimal Bases for Data Compression and for Statistical Estimation", Journal of Applied and Computer. Harmonic Analysis, Vol. 1, No. 1, pp. 100-115, 1993.

[16] Wim Sweldons, "The Lifting Scheme: A Construction of Second Generation Wavelets", Society for Industrial and Applied Mathematics, Vol. 29, No. 2, pp. 511-546, 2006.

[17] L. Anderson, N. Hall, B. Jawerth and G. Peters, "Wavelets on Closed Subsets of the Real Line", Recent Advances in Wavelet Analysis, pp. 1-61, 1993.

[18] P. Auscher, "Wavelets with Boundary Conditions on the Interval", Wavelets: A Tutorial in Theory and Applications, 1992.

[19] Charles K. Chui and Ewald Quak, "Wavelets on a Bounded Interval", Numerical Methods of Approximation Theory, Vol. 9, pp. 53-75, 1992.

[20] A. Cohen, I. Daubechies, B. Jawerth and P. Vial, "MultiResolution Analysis, Wavelets and Fast Algorithms on an Interval", Proceedings of the Academy of Sciences. Series I, Mathematics, Vol. 316, No. 5, pp. 417-421, 1993.

[21] A. Cohen, I. Daubechies and P. Vial "Multi-Resolution Analysis, Wavelets and Fast Algorithms on an Interval", Journal of Applied and Computer Harmonic Analysis, Vol. 1, No. 1, pp. 54-81, 1993.

[22] A. Cohen, W. Dahmen and R. DeVore, "Multi Scale Decompositions on Bounded Domains", Transactions of the American Mathematical Society, Vol. 352, No. 8, pp. 36513685, 2000. 
[23] M.D. Buhmann and C.A. Micchelli, "Spline Pre-Wavelets for Non-Uniform Knots", Numerical. Mathematics, Vol. 61, No. 1, pp. 455-474, 1992.

[24] W. Dehmen and C.A. Micchelli, "Banded Matrices with Banded Inverses II: Locally Finite Decompositions of the Spline Spaces", Constructive Approximation, Vol. 9, No. 23, pp. 263-281, 1993.

[25] C.K. Chui, "An Introduction to Wavelets", Academic Press, 1992.

[26] I. Daubechies, "Orthonormal Bases of Compactly Supported Wavelets II: Variations on a Theme", Journal on Mathematical Analysis, Vol. 24, No. 2, pp. 449-519, 1993.
[27] S. Pittner, "Dyadic Ortho-normal Wavelet Bases and Related Possibilities for Optimal Analysis and Representation of One-Dimensional Signals", PhD Dissertation, Vienna University of Technology, 1994.

[28] J.S.R. Jang, C.T. Sun and E. Mizutani, "Neuro-Fuzzy and Soft Computing-A computational Approach to Learning and Machine Intelligence", Pearson, 1996.

[29] M. Sugeno and G.T. Kang, "Structure Identification of Fuzzy Model”, Fuzzy Sets and Systems, Vol. 28, No. 1, pp. 15-33, 1988 